\title{
Epigenetic regulation of IQGAP2 promotes ovarian cancer progression via activating Wnt/ $\beta$-catenin signaling
}

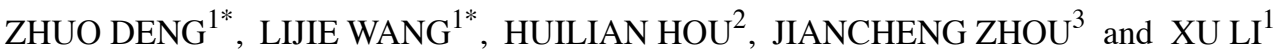 \\ ${ }^{1}$ Center for Translational Medicine, ${ }^{2}$ Department of Pathology, The First Affiliated Hospital of \\ Xi'an Jiaotong University College of Medicine, Xi'an 710061; ${ }^{3}$ Department of Urology, \\ Shaanxi Provincal People's Hospital, Xi'an 710068, P.R. China
}

Received July 28, 2015; Accepted September 18, 2015

DOI: $10.3892 /$ ijo.2015.3228

\begin{abstract}
Ovarian cancer is the most lethal gynecologic malignancy and most cases are diagnosed at an advanced stage with metastases; however, the molecular events supporting ovarian cancer development and progression remain poorly understood. In this study, by analysis of the genome-scale DNA methylation profiles of 8 healthy ovaries, 89 ovarian cancers and the corresponding 4 normal ovaries from The Cancer Genome Atlas, we unveiled the abnormalities in gene methylation of ovarian cancers, and found that IQGAP2 one of the most frequently altered genes, was significantly hypermethylated in ovarian cancer. There was an inverse correlation between IQGAP2 DNA methylation and mRNA expression, and IQGAP2 expression was downregulated in ovarian cancer. Further survival analysis indicated that decreased IQGAP2 was associated with a worse progression-free survival of patient with ovarian cancer, and biological function studies demonstrated that IQGAP2 inhibited ovarian cancer cell epithelial-mesenchymal transition, migration and invasion via suppression of Wnt-induced $\beta$-catenin nuclear translocation and transcriptional activity. Thus, these data identified IQGAP2 as a novel tumor suppressor for ovarian cancer to inhibit cell invasion through regulating $\mathrm{Wnt} / \beta$-catenin signaling, and provided a new biomarker and potential therapeutic strategy for this disease.
\end{abstract}

\section{Introduction}

Ovarian cancer is the most lethal gynecologic malignancy. Approximately $70 \%$ of cases are diagnosed at an advanced stage with metastases, and the 5-year survival of patients with

Correspondence to: $\mathrm{Dr} \mathrm{Xu} \mathrm{Li}$, Center for Translational Medicine, The First Affiliated Hospital of Xi'an Jiaotong University College of Medicine, 277 West Yanta Road, Xi'an 710061, P.R. China

E-mail: lixu1956@yahoo.com

*Contributed equally

Key words: IQGAP2, methylation, Wnt/ $\beta$-catenin, ovarian cancer, tumor progression advanced stage ovarian cancer is $<30 \%$ (1). The high rate of lethality from ovarian cancer is mainly due to the early progression of the diseases and lack of effective therapies for advanced ovarian cancers (2). Ovarian cancer displays highly molecular and genetic heterogeneity and complexity, although most cases of ovarian cancer are treated in a similar manner. A comprehensive understanding of molecular basis in ovarian cancer development and progression will help to identify better therapeutic strategies for this disease. Whole genome profiling of ovarian cancer has identified many dysregulated genes which are implicated in cell proliferation, invasion, motility, chromosomal instability, and gene silencing (3-5). However, the biological functions of these genes in ovarian cancer have not been fully studied.

IQ motif-containing GTPase activating protein 2(IQGAP2) belongs to the IQGAP family which contains a conventional Ras GTPase-activating protein (RasGAP) domain and an IQ motif $(6,7)$. The human IQGAP gene family consists of 3 members, IQGAP1, IQGAP2, and IQGAP3. IQGAP1 is the best characterized and is considered to be a scaffolding protein integrating diverse signaling pathways involved in cell proliferation, adhesion and migration $(8,9)$. Although IQGAP2 and IQGAP3 proteins exhibit a high level of sequence homology with IQGAP1, but their biological roles are poorly defined. Cumulative evidence from clinical specimens suggest IQGAP2 may be a tumor suppressor. Loss of IQGAP2 has been observed in gastric cancer, hepatocellular carcinoma and advanced prostate cancer (10-12), while the status of IQGAP2 in ovarian cancer remain unclear.

In the present study, by analysis of the differential epigenetic features between normal ovary and ovarian cancer, we identified that IQGAP2 was significantly downregulated in ovarian cancer due to elevated DNA methylation and was associated with patient prognosis. Biological function studies indicated that loss of IQGAP2 induced ovarian cancer cell epithelial-mesenchymal transition (EMT) and thus promoted cell migration and invasion. Further mechanism dissection demonstrated that loss of IQGAP2 potentiated Wnt/ $\beta$-catenin signaling rather than activating the Ras in ovarian cancer cells.

\section{Materials and methods}

Human ovarian cancer specimen. Five frozen human serous ovarian cancer and matched normal ovary specimens were 
obtained from patients who underwent surgical resection with the approval of the Institutional Review Board (IRB) of the First Hospital of Medical College of Xi'an Jiaotong University. Whole genomic DNA and total proteins were extracted for gene methylation and protein expression assays.

Bioinformatic analysis. HumanMethylation450 BeadChip arrays-based genome-scale DNA methylation data (Batch 9 and 40, update to February 4, 2014), RNA-Seq-based gene expression data for IQGAP2, and the clinical annotation data of serous ovarian cancer samples from The Cancer Genome Atlas (TCGA) were all retrieved through the CGDS server of the cBioportal hosted by the Memorial Sloan-Kettering Cancer Center. Gene microarray data for IQGAP2 of normal ovarian tissues and different subtypes of ovarian cancer tissues were retrieved from the GEO datasets (GSE26712, GSE6008). The $\mathrm{X}$-tile which is a bioinformatics tool for biomarker assessment and outcome-based cut-point optimization was used to generate an optimal cut-off point to dichotomize IQGAP2 mRNA level as 'High' and 'Low' using a Monte Carlo P-value $<0.05$.

Cell culture. Human ovarian cancer cell SKOV3 and SW626 were maintained in RPMI-1640 (Gibco, San Diego, CA, USA) medium supplemented with $10 \%$ fetal bovine serum (FBS), CAOV3 was maintained in Dulbecco's modified Eagle's medium (DMEM; Gibco) supplemented with FBS. All cells were cultured in a humidified incubator at $37^{\circ} \mathrm{C}$ with $5 \% \mathrm{CO}_{2}$.

Methylation specific PCR (MS-PCR). Genomic DNA from normal and ovarian cancer tissues was extracted using DNAse kit (Qiagen, Hilden, Germany), $2 \mu \mathrm{g}$ of genomic DNA was denatured by $\mathrm{NaOH}$ and modified by sodium bisulfite, and the modified DNA was purified using a Wizard DNA clean-up system (Promega, Madison, WI, USA). Bisulfite-treated DNA was then amplified as described (10). Briefly, PCR was performed by using methylation specific or unmethylation specific primers. Primers used for methylated IQGAP2 were: 5'-GGAGTGGGTCGTAGATTTTCGGGC-3' (sense) and 5'-CTACCCTCGCTAACCAAACTCGCG-3' (antisense), and primers used for unmethylated IQGAP2 were: 5'-AGGGA GTGGGTTGTAGATTTTTGGGT-3' (sense) and 5'-CAAC TACCCTCACTAACCAAACTCACA-3' (antisense). The PCR reaction was performed for 35 cycles of $95^{\circ} \mathrm{C}$ for $30 \mathrm{sec}, 58^{\circ} \mathrm{C}$ for $30 \mathrm{sec}$ and $72^{\circ} \mathrm{C}$ for $30 \mathrm{sec}$.

Reverse transcriptional $(R T)$ real-time PCR. Cells were treated with control (dimethyl sulfoxide, DMSO) or the indicated dose of methylation inhibitor 5-aza-2'-deoxycytidine (5-Aza; Sigma, St. Louis, MO, USA) for 7 days. Total RNA was extracted and $1 \mu \mathrm{g}$ RNA was reverse transcribed with a cDNA synthesis kit (Invitrogen). Real-time PCR analysis was set up with SYBR Green qPCR Supermix kit (Invitrogen) and carried out in the iCycler thermal cycler. The relative level of mRNA expression of each gene was determined by normalizing with $\beta$-actin. The primers used are: for $\beta$-actin, 5'-TACCACAGGCATTGTGATGG-3' (forward) and 5'-TTTGATGTCACGCACGATTT-3' (reverse); for IQGAP2, 5'-TCAAGATTGGACTGCTGGTG-3' (forward) and 5'-AGG TTTGGTCTGGAGGAGGT-3' (reverse).
Western blotting. Western blotting was performed as described (13). Briefly, cell lysates were harvested, equivalent amount of proteins were separated by $10 \%$ sodium dodecyl sulfate polyacrylamide gel electrophoresis and transferred to nitrocellulose membranes. Membranes were blocked with $5 \%$ skim milk, incubated with anti-IQGAP2 antibody (Santa Cruz, 1:500) overnight at $4{ }^{\circ} \mathrm{C}$ and followed by incubation with horseradish peroxidase conjugated secondary antibodies for 1 hour (h) at room temperature. Signals were then detected by chemiluminescence (Pierce, Rockford, IL, USA).

GST pull-down active Ras detection. Cells transiently transfected with different dose of IQGAP2 cDNA were then serum-starved for $12 \mathrm{~h}$. Active Ras (Raf1 binding Ras) was determined by the glutathione S-transferase (GST)-Raf1-RBD pull-down assay (Thermo Scientific, Waltham, MA, USA) followed by western blotting with Ras antibody. Briefly, cells were lysed in $500 \mu \mathrm{l}$ of lysis/binding buffer $(25 \mathrm{mM}$ Tris $\mathrm{HCl}, \mathrm{pH} 7.2,150 \mathrm{mM} \mathrm{NaCl}, 5 \mathrm{mM} \mathrm{MgCl}, 1 \% \mathrm{NP}-40$ and $5 \%$ glycerol) for $5 \mathrm{~min}$. Insoluble cellular debris was removed by centrifugation at $14,000 \mathrm{rpm}$ for $15 \mathrm{~min}$ at $4^{\circ} \mathrm{C}$. Cell lysate $(250 \mu \mathrm{g})$ was incubated with $80 \mu \mathrm{g}$ of GST-Raf1-RBD protein fused to glutathione agarose resin at $4^{\circ} \mathrm{C}$ for $60 \mathrm{~min}$. Cell lysate incubated with GTP $\gamma \mathrm{S}$ or GDP were set up as positive or negative control. After the incubation, samples were washed three times, resuspended in $30 \mu 1$ of SDS sample buffer and heated for $10 \mathrm{~min}$. Active Ras was detected by western blotting probed with Ras antibody.

Dual-luciferase reporter assay. $\beta$-catenin reporter gene luciferase assay was performed as described previously (14). Cells seeded in 24-well plates were transfected with $1 \mu \mathrm{g} \beta$-catenin firefly responsive luciferase constructs TOP and 2 ng Renilla luciferase construct as internal control. Forty-eight hours after transfection, TOP luciferase activity was measured using dual luciferase assay kit according to the manufacturer's protocol (Promega).

Immunofluorescence $(I F)$. The cells were fixed with $4 \%$ paraformaldehyde for $20 \mathrm{~min}$, permeabilized with $0.1 \%$ Triton X-100 and blocked with $3 \%$ bovine serum albumin for $1 \mathrm{~h}$. Cells were then incubated with $\beta$-catenin primary antibody (Cell Signaling Technology, 1:200) overnight at $4^{\circ} \mathrm{C}$ followed by TRITC labeled conjugates (Sigma; 1:500). Cells were counterstaining with 4,6-diamidino-2-phenylindole and fluorescence was visualized by fluorescence microscopy (Olympus Optical Co., Japan).

Transfection. Cells $\left(2 \times 10^{5}\right)$ at $60 \%$ confluence were seeded in a 6-well plate prior to the transfection, IQGAP2 overexpressing vector (pcDNA3-IQGAP2) and vector control (VC) were transfected into cells by $\mathrm{Xfect}^{\mathrm{TM}}$ Transfection reagent (Clontech, Palo Alto, CA, USA), $48 \mathrm{~h}$ after the transfection, G418 was used to select stable clones. IQGAP2 siRNAs were from RiboBio (Guangzhou, China) and transfected by X-tremeGENE siRNA transfection reagent (Roche Diagnostics, Mannheim, Germany) according to the manufacturer's instructions.

Transwell invasion assay. Matrigel-coated Transwell was used to examine the invasive ability of cells. Briefly, $5 \times 10^{4}$ cells in 


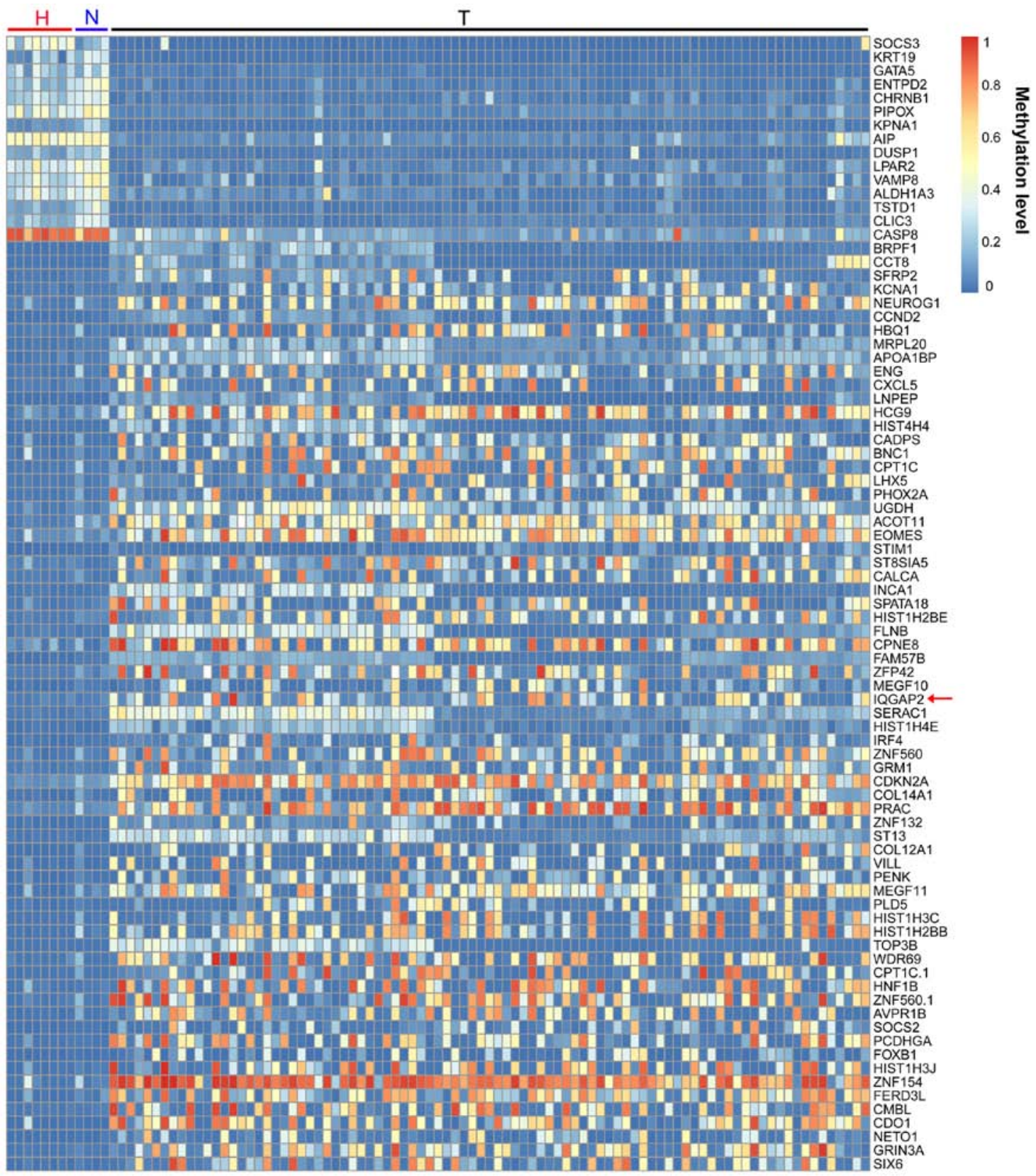

Figure 1. DNA methylation profiles of ovarian cancers $(T, n=89)$, corresponding normal ovaries $(N, n=4)$ and healthy ovaries $(H, n=8)$. Heat map showing the significantly hypomethylated (blue) and hypermethylated (red) genes.

$100 \mu \mathrm{l}$ of medium containing $0.5 \%$ FBS were seeded into the upper chamber, and $1 \mathrm{ml}$ of medium containing $20 \%$ FBS was added to the lower chamber. Cells in Transwell were then cultured at $37^{\circ} \mathrm{C}$ in $5 \% \mathrm{CO}_{2}$ for $48 \mathrm{~h}$. Cells on the lower surface of the membrane were stained with $0.5 \%$ crystal violent (Sigma) and photographed and counted.

Statistical analysis. The Kaplan-Meier method was used to analyze patient survival, and the log-rank test was used to assess the differences between groups. All the data from in vitro assay are presented as the mean \pm SEM from three independent experiments and the differences between two groups were compared by the Student's t-test. All statistical analyses were performed using GraphPad Prism 6.0 and SPSS16.0 software.

\section{Results}

IQGAP2 is hypermethylated in ovarian cancer. DNA methylation is an epigenetic mark which can be associated with transcriptional inactivity, it is essential for normal development (15) and has been implicated in many pathologies including cancer (16). We firstly analyzed the genome-scale DNA methylation profiles of 8 healthy ovary tissues, 89 ovarian cancers and the corresponding 4 normal ovary tissues from TCGA (17) to identify the altered gene methylation in ovarian cancers (Fig. 1). Within those differential genes, IQGAP2, a Ras GTPase-activating protein coding gene, was significantly hypermethylated in ovarian cancers $(\mathrm{P}<0.05$, Figs. 1 and $2 \mathrm{~A})$. To verify the data from TCGA, the methylation status of IQGAP2 in 5 serous ovarian cancers and the corresponding 
A
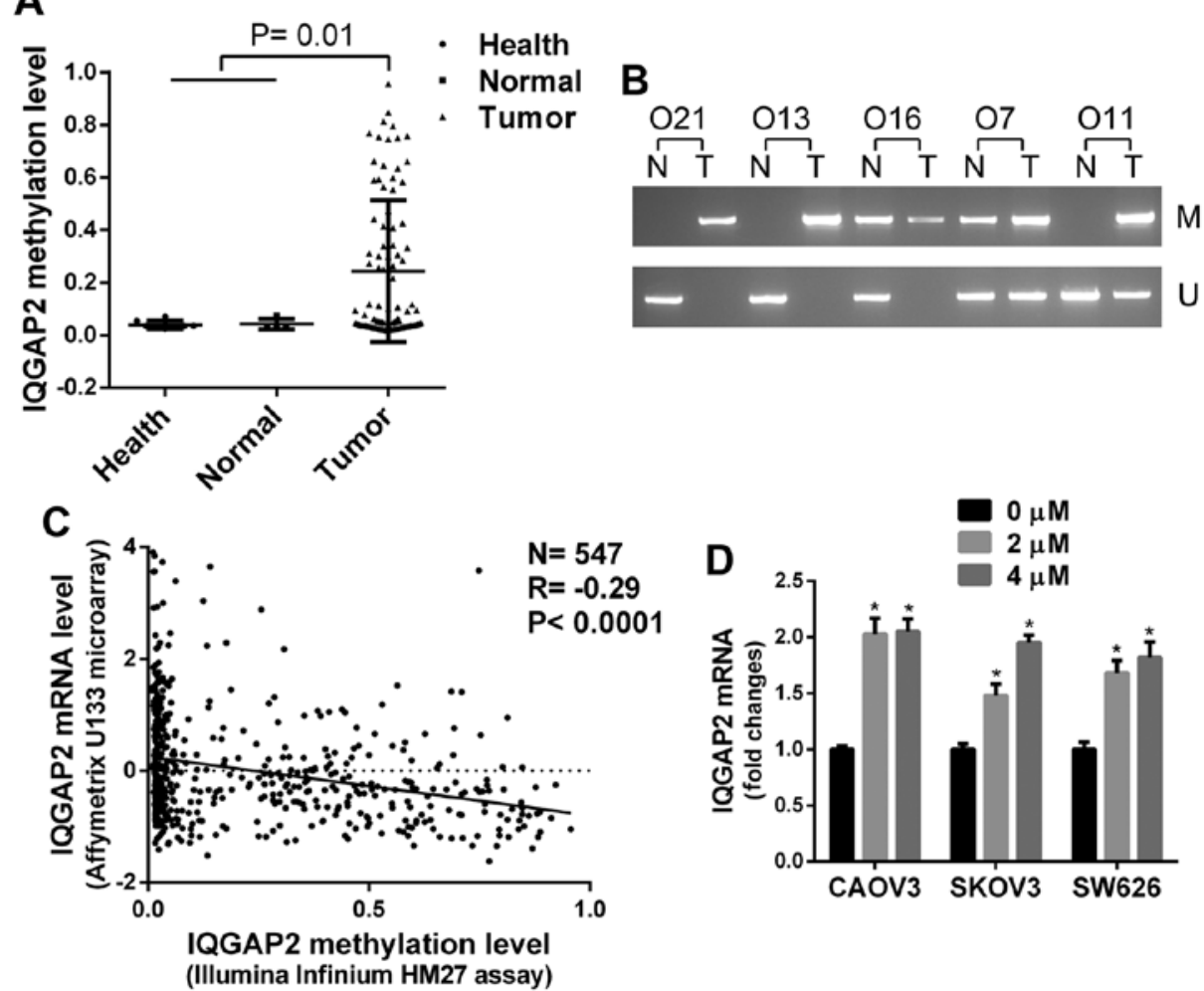

Figure 2. IQGAP2 is hypermethylated in ovarian cancers. (A) IQGAP2 methylation levels in ovarian cancers (n=89), corresponding normal ovaries (n=4) and health ovaries $(n=8)$. (B) MS-PCR showing methylated (M) and unmethylated (U) IQGAP2 in serous ovarian cancers. (C) Pearson correlation analysis of IQGAP2 DNA methylation and mRNA levels in serous ovarian cancers. (D) RT-PCR showing IQGAP2 expression in ovarian cancer cells treated at different doses of methylation inhibitor 5-Aza.

normal ovary tissues from an independent cohort was examined by MS-PCR. Four of 5 ovarian cancers exhibited increased methylation of IQGAP2 compared to normal tissues (Fig. 2B).

To determine whether aberrant methylation results in inactivation of IQGAP2 in ovarian cancer, the association between IQGAP2 DNA methylation and mRNA expression in 547 serous ovarian cancers from TCGA was analyzed, and a reverse correlation was found $(\mathrm{P}<0.001$, Fig. 2C). Additionally, ovarian cancer cells were treated with a DNA methylation inhibitor, 5-Aza, and the expression of IQGAP2 in cells were significantly induced (Fig. 2D). Taken together, these data indicate that IQGAP2 is hypermethylated in ovarian cancer.

IQGAP2 is downregulated in ovarian cancer and associated with a poor survival of patient. We further analyzed the expression of IQGAP2 in 10 normal ovary and 185 ovarian cancer tissues from GEO database and found IQGAP2 mRNA was significantly decreased in ovarian cancers $(P=0.0006$, Fig. 3A). When subtypes were stratified, serous and clear cell ovarian cancers showed decreased IQGAP2 mRNA expression $(\mathrm{P}<0.05)$, while there was no significant difference in IQGAP2 level between endometrioid and normal tissues $(\mathrm{P}=0.079$, Fig. 3B). In addition, the expression of IQGAP2 protein was examined in those 5 serous ovarian cancers, 2 of them showed significantly decreased IQGAP2 expression compared to the corresponding normal ovary tissues (Fig. 3C). Furthermore, to determine whether IQGAP2 expression is a prognostic factor for ovarian cancer, Kaplan-Meier (log-rank test) survival assay was performed to determine the association between IQGAP2 level and patient survival from TCGA. The data indicated that although IQGAP2 was not correlated with patient overall survival $(\mathrm{P}=0.718)$, decreased IQGAP2 mRNA was correlated with a worse progression-free survival of ovarian cancer patients $(\mathrm{P}=0.045$, Fig. 3D).

IQGAP2 negatively regulates the invasiveness of ovarian cancer. To determine the biological function of IQGAP2 in ovarian cancer, stable IQGAP2-overexpressing (IQGAP2) and vector control (VC) cells from SKOV3, and IQGAP2 silencing (si-IQGAP2) and control (Scramble) cells from SW626 were established. In vitro cell migration and invasion assay demonstrated that overexpression of IQGAP2 suppressed while loss of IQGAP2 promoted the migration and invasion of ovarian cancer cells (Fig. 4A and B). EMT is a process thought to initiate metastasis by enhancing the motility of tumor cells (18). We examined the expression of EMT markers in IQGAP2-overexpressing or IQGAP2-silenced cells, and the results showed that IQGAP2 upregulated epithelial markers, such as E-cadherin and CK-18, and downregulated mesenchymal makers, such as vimentin, MMP-2 and MMP-9 (Fig. 4C). These data indicate IQGAP2 as a suppressor for the invasiveness of ovarian cancer cells.

IQGAP2 suppresses cell invasiveness via inhibition of $W n t / \beta$ catenin signaling. To investigate the mechanism of IQGAP2 in the regulation of cell invasiveness, we firstly determined the effect of IQGAP2 on the activity of Ras in ovarian cancer 
B GSE6008
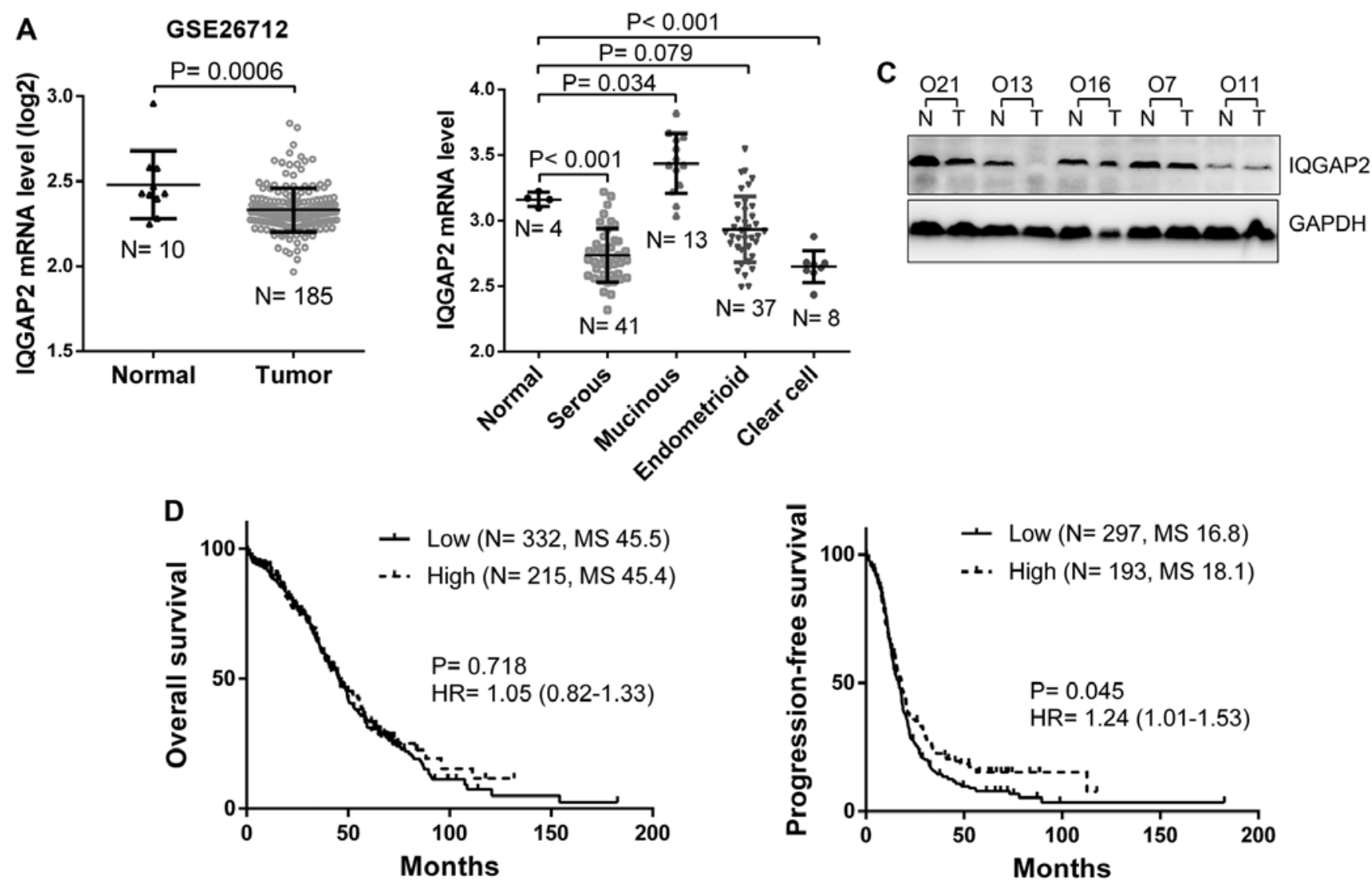

Figure 3. Downregulated IQGAP2 is associated with poor survival of ovarian cancer. (A and B) IQGAP2 mRNA levels in normal and ovarian cancer tissues. (C) Western blotting showing IQGAP2 protein expression in normal and serous ovarian cancer tissues. (D) Kaplan-Meier analysis (log-rank test) showing the associations between IQGAP2 level and the overall or progression-free survival of patients with serous ovarian cancer.

cells. SKOV3 cells were transfected with different doses of IQGAP2 cDNA and GST pull-down active Ras assay demonstrated that the activity of Ras in cells were not affected by IQGAP2 (Fig. 5A). We then determined the activities of ERK, AKT and $\beta$-catenin signaling which have been reported to be regulated by IQGAP2 in other cancers $(11,12)$, and found that IQGAP2 significantly suppressed $\beta$-catenin protein expression as well as its nuclear translocation (Fig. 5B and C). Notably, IQGAP2 was also able to inhibit Wnt3ainduced transcriptional activity of $\beta$-catenin (Fig. 5D), which indicated that IQGAP2 was a suppressor of $\mathrm{Wnt} / \beta$-catenin signaling. We further found that overexpression of IQGAP2 could ablate Wnt3a-induced cell invasion (Fig. 5E). To determine whether the inactivation of $\beta$-catenin is a critical for IQGAP2-regulated cell invasion, IQGAP2-overexpressing SKOV3 cells were transfected with a constitutively activated $\beta$-catenin ( $\beta$-catenin $\mathrm{CA}$ ), and the results showed that $\beta$-catenin CA significantly rescued the invasiveness of cells (Fig. 5F). Collectively, these data indicate that IQGAP2 suppresses ovarian cancer cell invasiveness via the inhibition of $\mathrm{Wnt} / \beta$-catenin signaling.

\section{Discussion}

While the molecular basis for ovarian cancer remains poorly defined, studies suggest many gene alterations due to muta- tion, copy number variation or epigenetic abnormalities may contribute to this disease. The well-known oncogenes and tumor suppressors in ovarian cancer include TP53, PIK3C, $B R C A 1$ and BRCA2 $(19,20)$. In particular, TP53 is the most common mutated gene occurring in $>70 \%$ of the advanced cases. More recently, along with the improvement of whole genomic analysis tools, other genes have also been identified, such as EMSY, PTEN, RAD5IC and CTTNBI (21). DNA methylation is associated with gene transcription silencing, dysregulated gene methylation has been implicated in almost all types of cancer (16). We compared gene methylation level between healthy/normal ovaries and ovarian cancers, and found that many critical proto-oncogenes and antioncogenes such as BRAF1, SIX6, FOXB1, SFRP2, CDKN2A and CASP 8 are dysregulated by DNA methylation in ovarian cancers. Functions of these genes in ovarian cancer need to be further defined. Among those altered genes, IQGAP2 is hypermethylated and its expression is decreased in ovarian cancers. Indeed, loss of heterozygosity of IQGAP2 is reported in ovarian cancer (22). Hypermethylation of IQGAP2 is also observed in gastric cancer (10). Notably, we found decreased IQGAP2 level is associated with a poor survival of patient, indicating IQGAP2 as a new prognostic marker for ovarian cancer.

The Ras pathway is one of the most commonly deregulated pathways in human cancer. Hyperactivation of Ras 

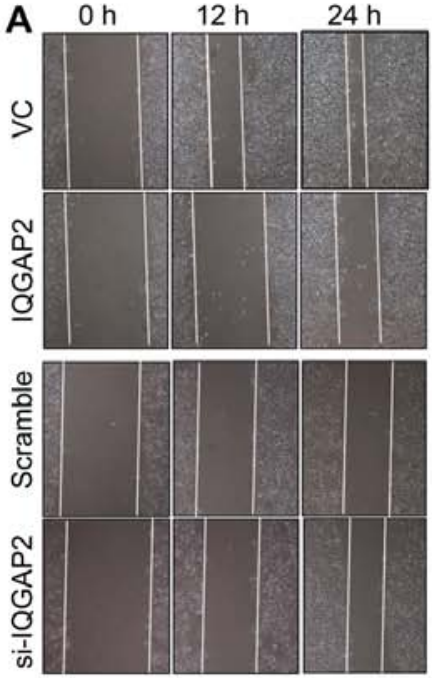
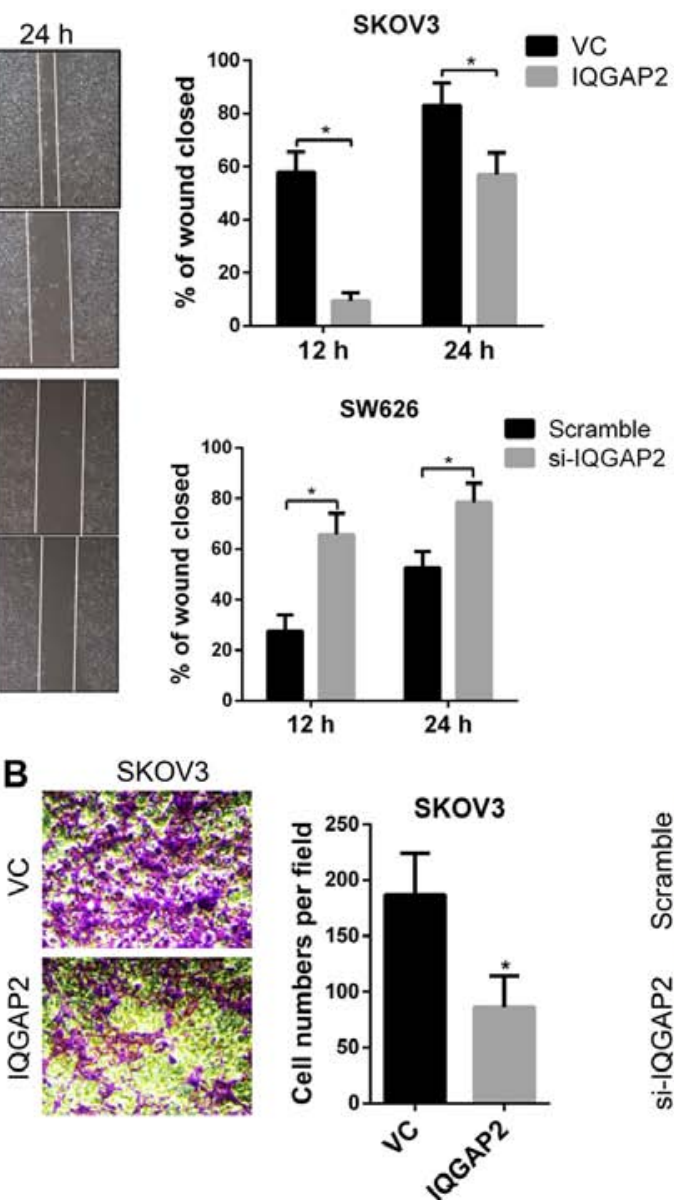

C

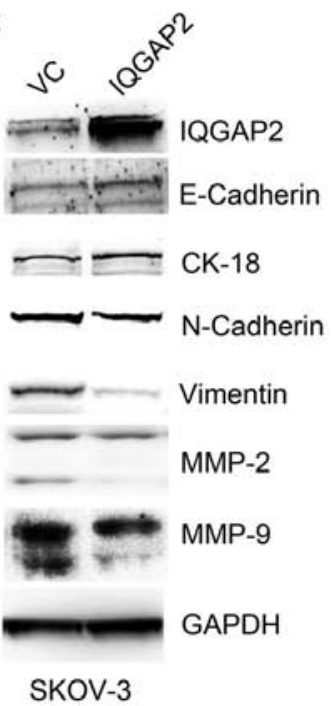

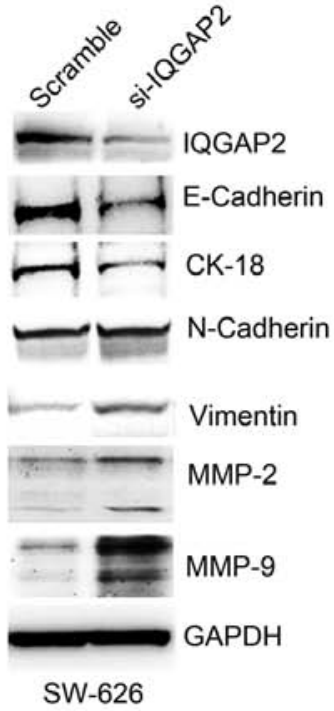

SW626
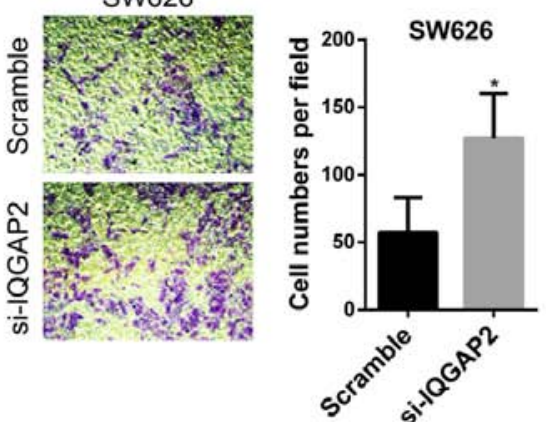

Figure 4. IQGAP2 negatively regulates the invasiveness of ovarian cancer. Ovarian cancer SKOV-3 cell was overexpressed with control vector (VC) and IQGAP2 cDNA, SW626 was transfected with scramble RNA and IQGAP2 siRNA. (A) Wound healing assay showing cell migration, (B) Transwell assay showing cell invasion, and $(\mathrm{C})$ western blotting showing gene expression in the indicated cells.

A
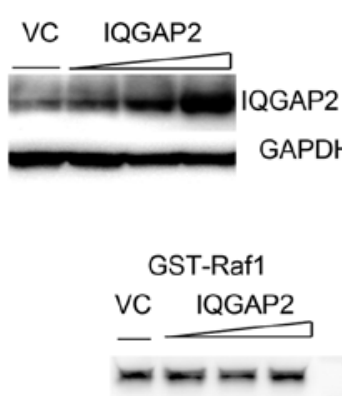

D

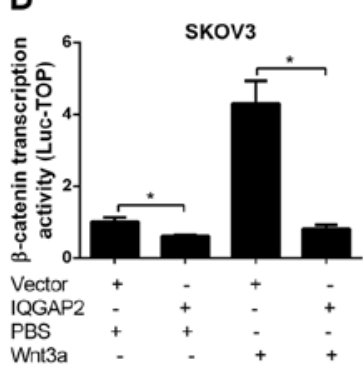

B SKOV3 sw626

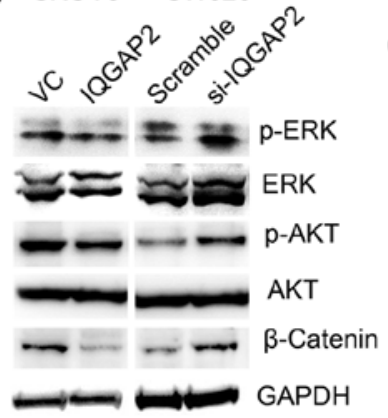

C $\quad \mathrm{SKOV}_{3}$

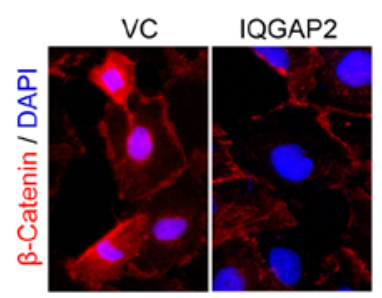

E

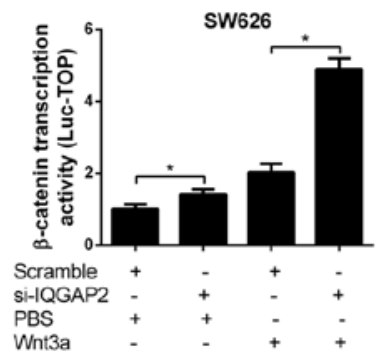

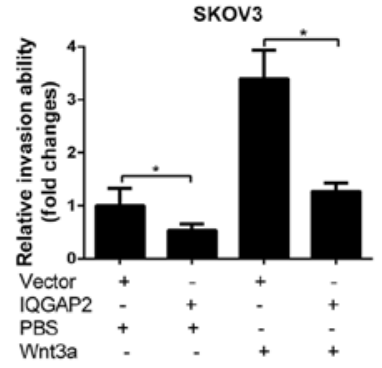

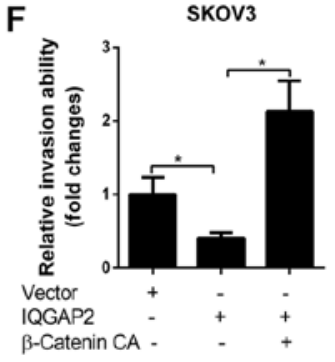

Figure 5. IQGAP2 suppresses cell invasiveness via inhibiting Wnt/ $\beta$-catenin signaling. (A) SKOV3 cells were transfected with different doses of IQGAP2 cDNA, Ras activity was determined by GST-Raf1 pull-down assay. GTP and GDP treatment was respectively used as positive and negative control. (B) Western blotting showing ERK, AKT and $\beta$-catenin activity in cells transfected with IQGAP2 cDNA or siRNA. (C) Immunofluorescence (IF) showing $\beta$-catenin localization in SKOV3 cells transfected with VC or IQGAP2. (D) Luciferase assay showing $\beta$-catenin transcriptional activity in cells treated with IQGAP2 cDNA or siRNA in combination with Wnt3a. (E) Invasive ability of SKOV3 cells treated with IQGAP2 cDNA in combination of Wnt3a. (F) Invasive ability of SKOV3 cells treated with IQGAP2 cDNA in combination with constitutively activated $\beta$-catenin cDNA ( $\beta$-catenin CA). 
pathway can be caused by mutations in Ras genes or loss of RasGAP proteins (23). There are 14 predicted RasGAP genes in the human genome and they all contain a RasGAP domain but share little similarity in other regions. Except for a RasGAP domain, the IQGAP subfamily also contain an actin-binding calponin homology domain $(\mathrm{CH})$, a polyproline-binding domain (WW) and four IQ calmodulinbinding motifs (IQ) (24). IQGAP1 functions as a scaffolding protein that is required for RAS-driven tumorigenesis and metastasis, many studies indicate a positive role for IQGAP1 in cancer. It modulates MAPK signaling pathway to regulate cell proliferation and differentiation and interacts with $\beta$-catenin and E-cadherin to regulate intercellular adhesion and migration $(25,26)$. Although IQGAP2 shares similarity in sequencing with IQGAP1, it exhibits opposite roles and functions as a tumor suppressor. Deficiency of IQGAP2 leads to the development of hepatocellular carcinoma (27). IQGAP2 is abnormally methylated in gastric cancers and is significantly associated with tumor invasion and a poor prognosis (10). IQGAP2 is lost in advanced prostate cancers and inhibits EMT and attenuates serum induced AKT activation (12). We found IQGAP2 is downregulated in ovarian cancers and suppresses cell migration and invasion. Mechanistically, loss of IQGAP2 is able to activate ERK, AKT and Wnt/ $\beta$-catenin signaling pathways, but it fails to affect the activity of Ras. This suggests that IQGAP2 does not exhibit RasGAP activity in ovarian cancer cells. In particular, Wnt/ $\beta$-catenin seems to be the critical mediator for IQGAP2-regulated migration and invasion in ovarian cancer cells. In hepatocytes, IQGAP2 interacts with $\beta$-catenin and anchors $\beta$-catenin at the submembrane region along with E-cadherin, it also, as a part of the $\beta$-catenin destruction complex, consists of GSK3 $\beta$ kinase, Axin and adenomatous polyposis coli (APC) (28). We demonstrated that IQGAP2 promotes $\beta$-catenin cytosol localization and thus suppresses its transcriptional activity.

Many patients with ovarian cancer exhibit early extension of tumors to the outside of ovaries at the time of diagnosis. EMT is a process characterized by the gain of mesenchymal markers (e.g., N-cadherin, vimentin) and the loss of epithelial markers (e.g., E-cadherin, cytokeratin), as well as altered morphological features associated with this process inducing increased motility and invasion of cancer cells (29). EMT occurs during ovarian cancer progression, however, the underlying mechanisms are not well established. The Wnt/ $\beta$ catenin signaling pathway has been reported to induce EMT in ovarian cancer and other tumor cells $(30,31)$. In the present study, we demonstrate a novel role of IQGAP2 in suppressing ovarian cancer EMT through regulating Wnt $\beta$ catenin signaling, providing a new biomarker and potential therapeutic strategy for ovarian cancer.

\section{References}

1. Siegel R, Ma J, Zou Z and Jemal A: Cancer statistics, 2014. CA Cancer J Clin 64: 9-29, 2014.

2. Berek JS, Crum C and Friedlander M: Cancer of the ovary, fallopian tube, and peritoneum. Int J Gynaecol Obstet 119 (Suppl 2): S118-S129, 2012.

3. Quitadamo A, Tian L, Hall B and Shi X: An integrated network of microRNA and gene expression in ovarian cancer. BMC Bioinformatics 16 (Suppl 5): S5, 2015.
4. Ying H, Lv J, Ying T, Jin S, Shao J, Wang L, Xu H, Yuan B and Yang Q: Retraction note: Gene-gene interaction network analysis of ovarian cancer using TCGA data. J Ovarian Res 8: $18,2015$.

5. Shah RH, Scott SN, Brannon AR, Levine DA, Lin O and Berger MF: Comprehensive mutation profiling by next-generation sequencing of effusion fluids from patients with high-grade serous ovarian carcinoma. Cancer Cytopathol 123: 289-297, 2015.

6. Briggs MW and Sacks DB: IQGAP proteins are integral components of cytoskeletal regulation. EMBO Rep 4: 571-574, 2003.

7. Maertens $\mathrm{O}$ and Cichowski $\mathrm{K}$ : An expanding role for RAS GTPase activating proteins (RAS GAPs) in cancer. Adv Biol Regul 55: 1-14, 2014.

8. Yamaoka-Tojo M, Ushio-Fukai M, Hilenski L, Dikalov SI, Chen YE, Tojo T, Fukai T, Fujimoto M, Patrushev NA, Wang N, et al: IQGAP1, a novel vascular endothelial growth factor receptor binding protein, is involved in reactive oxygen species - dependent endothelial migration and proliferation. Circ Res 95: 276-283, 2004.

9. Katata T, Irie K, Fukuhara A, Kawakatsu T, Yamada A, Shimizu K and Takai Y: Involvement of nectin in the localization of IQGAP1 at the cell-cell adhesion sites through the actin cytoskeleton in Madin-Darby canine kidney cells. Oncogene 22: 2097-2109, 2003.

10. Jin SH, Akiyama Y, Fukamachi H, Yanagihara K, Akashi T and Yuasa Y: IQGAP2 inactivation through aberrant promoter methylation and promotion of invasion in gastric cancer cells. Int J Cancer 122: 1040-1046, 2008.

11. White CD, Khurana H, Gnatenko DV, Li Z, Odze RD, Sacks DB and Schmidt VA: IQGAP1 and IQGAP2 are reciprocally altered in hepatocellular carcinoma. BMC Gastroenterol 10: $125,2010$.

12. Xie Y, Yan J, Cutz JC, Rybak AP, He L, Wei F, Kapoor A, Schmidt VA, Tao L and Tang D: IQGAP2, A candidate tumour suppressor of prostate tumorigenesis. Biochim Biophys Acta 1822: 875-884, 2012

13. Zhou J, Deng Z, Chen Y, Gao Y, Wu D, Zhu G, Li L, Song W, Wang X, Wu K, et al: Overexpression of FABP7 promotes cell growth and predicts poor prognosis of clear cell renal cell carcinoma. Urol Oncol 33: 113.e9-113.e17, 2015.

14. Zhou J, Wu K, Gao D, Zhu G, Wu D, Wang X, Chen Y, Du Y, Song W, Ma Z, et al: Reciprocal regulation of hypoxia-inducible factor $2 \alpha$ and GLI1 expression associated with the radioresistance of renal cell carcinoma. Int J Radiat Oncol Biol Phys 90: 942-951, 2014.

15. Reik W: Stability and flexibility of epigenetic gene regulation in mammalian development. Nature 447: 425-432, 2007.

16. Jones PA and Baylin SB: The epigenomics of cancer. Cell 128: 683-692, 2007.

17. Cerami E, Gao J, Dogrusoz U, Gross BE, Sumer SO, Aksoy BA, Jacobsen A, Byrne CJ, Heuer ML, Larsson E, et al: The cBio cancer genomics portal: An open platform for exploring multidimensional cancer genomics data. Cancer Discov 2: 401-404, 2012.

18. Kalluri R and Weinberg RA: The basics of epithelial-mesenchymal transition. J Clin Invest 119: 1420-1428, 2009.

19. Sowter HM and Ashworth A: BRCA1 and BRCA2 as ovarian cancer susceptibility genes. Carcinogenesis 26: 1651-1656, 2005.

20. Kohler MF, Kerns BJ, Humphrey PA, Marks JR, Bast RC Jr and Berchuck A: Mutation and overexpression of p53 in early-stage epithelial ovarian cancer. Obstet Gynecol 81: 643-650, 1993.

21. Bell D, Berchuck A, Birrer M, Chien J, Cramer DW, Dao F, Dhir R, DiSaia P, Gabra H, Glenn P, et al; Cancer Genome Atlas Research Network: Integrated genomic analyses of ovarian carcinoma. Nature 474: 609-615, 2011.

22. Gorringe KL, Ramakrishna M, Williams LH, Sridhar A, Boyle SE, Bearfoot JL, Li J, Anglesio MS and Campbell IG: Are there any more ovarian tumor suppressor genes? A new perspective using ultra high-resolution copy number and loss of heterozygosity analysis. Genes Chromosomes Cancer 48: 931-942, 2009.

23. Karnoub AE and Weinberg RA: Ras oncogenes: Split personalities. Nat Rev Mol Cell Biol 9: 517-531, 2008.

24. Bernards A and Settleman J: GAP control: Regulating the regulators of small GTPases. Trends Cell Biol 14: 377-385, 2004.

25. Fukata M, Watanabe T, Noritake J, Nakagawa M, Yamaga M, Kuroda S, Matsuura Y, Iwamatsu A, Perez F and Kaibuchi K: Rac1 and Cdc42 capture microtubules through IQGAP1 and CLIP-170. Cell 109: 873-885, 2002. 
26. Roy M, Li Z and Sacks DB: IQGAP1 is a scaffold for mitogenactivated protein kinase signaling. Mol Cell Biol 25: 7940-7952, 2005.

27. Schmidt VA, Chiariello CS, Capilla E, Miller F and Bahou WF: Development of hepatocellular carcinoma in Iqgap2-deficient mice is IQGAP1 dependent. Mol Cell Biol 28: 1489-1502, 2008.

28. Schmidt VA: Watch the GAP: Emerging roles for IQ motifcontaining GTPase-activating proteins IQGAPs in hepatocellular carcinoma. Int J Hepatol 2012: 958673, 2012.

29. Kang Y and Massagué J: Epithelial-mesenchymal transitions: Twist in development and metastasis. Cell 118: 277-279, 2004.
30. Yoshioka S, King ML, Ran S, Okuda H, MacLean JA II, McAsey ME, Sugino N, Brard L, Watabe K and Hayashi K: WNT7A regulates tumor growth and progression in ovarian cancer through the WNT/ $\beta$-catenin pathway. Mol Cancer Res 10: 469-482, 2012.

31. Wu Y, Ginther C, Kim J, Mosher N, Chung S, Slamon D and Vadgama JV: Expression of Wnt3 activates Wnt $/ \beta$-catenin pathway and promotes EMT-like phenotype in trastuzumabresistant HER2-overexpressing breast cancer cells. Mol Cancer Res 10: 1597-1606, 2012. 